\title{
Reliability Assessment of Competing Risks with Generalized Mixed Shock Models
}

\author{
Koosha Rafiee $^{1}$, Qianmei Feng ${ }^{1,2^{*}}$, David W. Coit ${ }^{3}$ \\ ${ }^{1}$ Department of Industrial Engineering, University of Houston, Houston, TX 77204 \\ ${ }^{2}$ College of Mechanical Automation, Wuhan University of Science and Technology, Hubei, China \\ ${ }^{3}$ Department of Industrial \& Systems Engineering, Rutgers University, Piscataway, NJ, 08854
}

\begin{abstract}
This paper investigates reliability modeling for systems subject to dependent competing risks considering the impact from a new generalized mixed shock model. Two dependent competing risks are soft failure due to a degradation process, and hard failure due to random shocks. The shock process contains fatal shocks that can cause hard failure instantaneously, and nonfatal shocks that impact the system in three different ways: 1) damaging the unit by immediately increasing the degradation level, 2) speeding up the deterioration by accelerating the degradation rate, and 3) weakening the unit strength by reducing the hard failure threshold. While the first impact from nonfatal shocks comes from each individual shock, the other two impacts are realized when the condition for a new generalized mixed shock model is satisfied. Unlike most existing mixed shock models that consider a combination of two shock patterns, our new generalized mixed shock model includes three classic shock patterns. According to the proposed generalized mixed shock model, the degradation rate and the hard failure threshold can simultaneously shift multiple times, whenever the condition for one of these three shock patterns is satisfied. An example using micro-electro-mechanical systems devices illustrates the effectiveness of the proposed approach with sensitivity analysis.
\end{abstract}

Keywords: Dependent competing risks, changing degradation rate, shifting hard failure threshold, generalized mixed shock model

*Corresponding author. Email: qmfeng@uh.edu; Telephone: 1-713-743-2870

(C) 2016. This manuscript version is made available under the Elsevier user license http://www.elsevier.com/open-access/userlicense/1.0/ 


\section{Introduction}

Complex systems may experience multiple failure processes that compete against each other, and whichever occurs first can cause the system to fail. Two major failure processes are related to degradation processes and random shocks. Systems, especially mechanical devices, usually deteriorate and lose their intended functionality due to wear, fatigue, erosion, corrosion, and aging (Huynh et al. 2012). Systems can also stop functioning abruptly due to shocks, overloads, and other external stresses. For example, a battery that supplies electrical power by chemical reaction weakens during usage. On the other hand, a battery can suddenly fail under unusual environmental conditions or stresses, such as overheating and over-voltage. These competing risks can be either independent or dependent. Previously, most papers assumed that the degradation process and the shock process are independent (Li \& Pham 2005; Chien et al. 2006; van der Weide et al. 2010), which may not always be a realistic assumption. For instance, shocks can naturally impact the degradation process due to material properties and structures. Therefore, the dependence between these failure processes should not be neglected in reliability analysis of complex systems.

Recently, there have been many studies devoted to reliability analysis for systems subject to multiple dependent competing failure processes (Wang et al. 2008; Lehmann 2009; Jiang et al. 2012). For complex systems such as micro-electro-mechanical systems (MEMS), the dependence between the two competing risks of degradation and shocks can lead to complex behaviors, which can be considered in a variety of different analytical perspectives. When a system withstands shocks, it becomes more vulnerable to upcoming shocks, while at the same time, the shock process can also damage the system and accelerate the deterioration process (Tanner et al. 2000). This paper aims to extend the previous models (Jiang et al. 2012; Rafiee et al. 2014a, 
2014b) by incorporating multiple sources of dependence between competing risks into a rich reliability model.

We propose a new reliability model for systems subject to dependent competing risks under the impact from a generalized mixed shock model. Unlike most existing mixed shock models that consider a combination of two shock patterns, our generalized mixed shock model includes three classic shock patterns: extreme shock model, $\delta$-shock model and run shock model. The condition for our generalized mixed shock model is satisfied when any of the three classic shock patterns occurs, i.e., the condition for the generalized mixed shock model is satisfied as soon as $(i)$ there is a shock whose magnitude is greater than the extreme shock model threshold, (ii) there is a set of $n$ consecutive shocks whose magnitudes are greater than the run shock model threshold, or (iii) the time lag between two successive shocks is less than $\delta$ in the $\delta$-shock model.

The two dependent competing risks considered in this research are soft failure due to a degradation process, and hard failure due to shocks. In this paper, a shock process is considered to contain fatal shocks that can cause the hard failure instantaneously, and nonfatal shocks that simultaneously impact the system in three different ways: 1) damaging the unit by increasing the degradation level instantaneously, 2) speeding up the deterioration by accelerating the degradation rate, and 3) weakening the unit strength by reducing the hard failure threshold. While the first impact of nonfatal shocks comes from each individual shock, the other two impacts are realized when the condition for the generalized mixed shock model is satisfied. A series of random shocks can accelerate the deterioration process; at the same time, the system may become more vulnerable to upcoming shocks leading to a reduced hard failure threshold. The proposed model is presented in Figure 1 by highlighting different impacts from random shocks. 


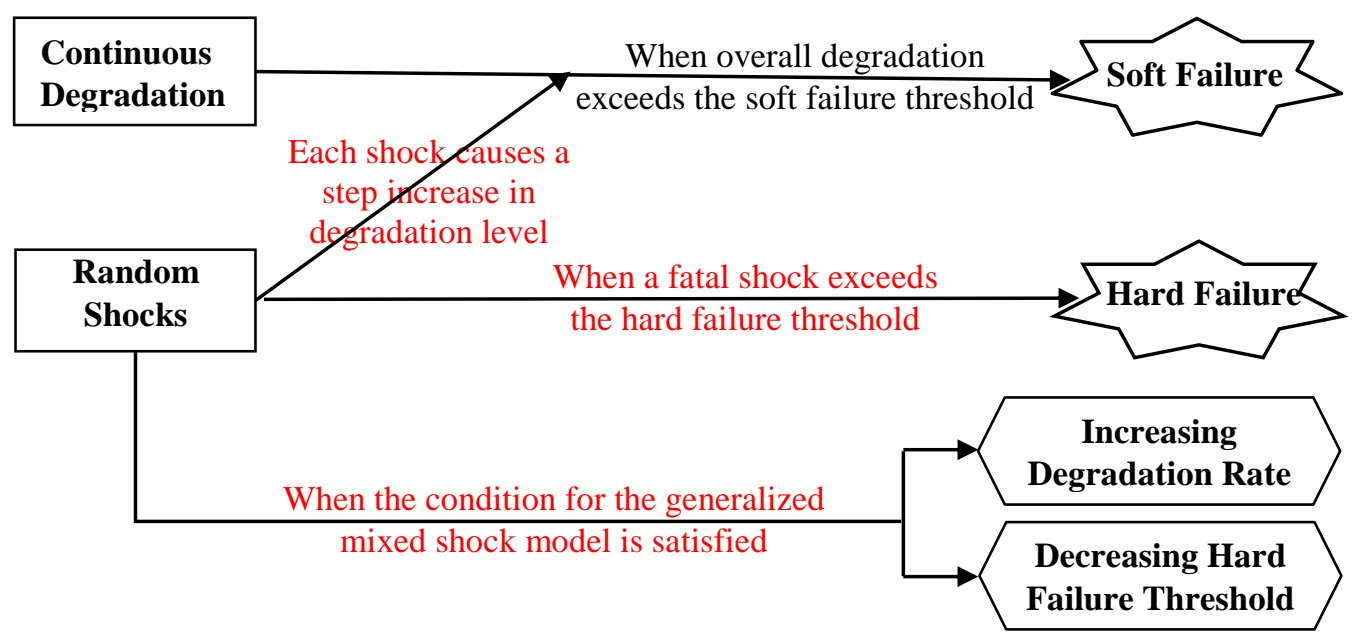

Figure 1: Impacts from random shocks in two failure processes

Compared to existing studies (Jiang et al. 2012; Rafiee et al. 2014a, 2014b), the main contributions of this paper are considered to be:

1. The degradation rate and the hard failure threshold can shift/transition simultaneously.

2. The transition in the degradation rate and the hard failure threshold is triggered by any of the three shock models in a new generalized mixed shock model, instead of only one shock model.

3. The transition in the degradation rate and the hard failure threshold can occur multiple times, whenever the condition for the generalized mixed shock model is satisfied.

This paper is organized as follows. Section 2 presents a brief literature review on multiple dependent failure processes and the motivation to conduct this research. Section 3 describes two dependent failure processes of hard failure and soft failure. In Section 4, we derive the reliability model for a system subject to a generalized mixed shock model. Section 5 presents a numerical example and evaluates the effects of model parameters. Section 6 summarizes the article and concluding remarks are made. 


\section{Literature Review}

External shocks are a major source leading to the failure of a device or an infrastructure. In the literature, there has been extensive research devoted to three typical shock models: extreme shock model, $\delta$-shock model, and run shock model. In the extreme shock model, a system fails as soon as the magnitude of any shock is greater than a threshold (Ye et al. 2011). In the $\delta$-shock model, a system is considered failed when the time lag of two successive shocks is less than a threshold $\delta$ (Li $\&$ Kong 2007). In the run shock model, a system breaks down after a run of $n$ consecutive shocks above a critical level (Mallor \& Omey 2001). A mixed shock model is a combination of two or more classic shock models (Gut 2001). Wang and Zhang (2005) analyzed the reliability for a system that fails due to a mixed shock model including the extreme shock model and the $\delta$-shock model. Bai et al. (2006) proposed a mixed shock model including extreme shock model, cumulative shock model, and $\delta$-shock model that can cause system failure immediately. Rafiee et al. (2014c) studied the reliability of systems that fail instantaneously if a shock belongs to any of three classic shock models (i.e., extreme shock model, run shock model, and $\delta$-shock model) in a mixed shock model. Unlike existing mixed shock models that can cause system failure, our new generalized mixed shock model (including extreme shock model, $\delta$ shock model, and run shock model) can trigger a shift/transition in both the degradation rate and the hard failure threshold.

Saassouh et al. (2007) proposed a competing-risk model with a changing degradation rate. The system degrades according to gamma process and the degradation rate increases as soon as the system transitions to the second mode. Li et al. (2010) worked on reliability modeling for a deteriorating system considering shock effects, when degradation processes are either independent or dependent. Huynh et al. (2011) worked on dependent failure processes 
when the arrival rate of random shocks depends on the degradation. Ye et al. (2011) investigated time-to-failure models by using Brown-Proschan model to obtain the degradation process and shock process, when no information about the degradation level and shock size is available.

Recent research has started to consider the impacts of shocks on the degradation process in dependent competing risks. Wang et al. (2011) investigated reliability modeling when the degradation and shocks are involved. Each shock impacts degradation in two forms: a sudden increase in the degradation rate, and an abrupt damage. Peng et al. (2011) proposed a reliability model for systems with two dependent failure processes: soft failure due to degradation that is accumulated by continuous degradation over time and sudden increase due to shocks, and hard failure caused by the same shocks. To extend the study in Peng et al. (2011), Jiang et al. (2012) considered the case where the system resistance to failure weakens when withstanding shocks. The hard failure threshold can shift to a lower level when one of the extreme shock, $m$-shock, and $\delta$-shock models occurs. Rafiee et al. (2014a) extended the model presented in Peng et al. (2011) by introducing new reliability models where the degradation rate can accelerate due to different shock patterns. As a result of withstanding shocks, the degradation rate is not a constant when the system becomes more prone to fatigue. By considering the declining hard failure threshold according to changes in degradation, Rafiee et al. (2014b) introduced reliability models for a device with two dependent failure processes: soft failure due to degradation, and hard failure due to random shocks.

However, none of the existing research considers the integration or combination of these impacts of shocks on a system in one general model. The new model proposed in this paper takes care of three different impacts of shocks on the system: 1) abrupt damage on degradation, 2) increasing the degradation rate, and 3) shifting the hard failure threshold. What distinguishes our 
model from the previous research lies on this new assumption that the degradation rate and the hard failure threshold can simultaneously change multiple times, given the condition for the generalized mixed shock model is satisfied. This general assumption reflects the more complex behavior associated with modern systems and structures that experience multiple sources of external shocks.

\section{Reliability Modeling with Generalized Mixed Shock Model}

Figure 2 depicts two dependent failure processes: soft failure due to a degradation process and hard failure due to random shocks. A soft failure occurs when the overall degradation level exceeds a predetermined threshold $H$. The continuous degradation by time $t$, $X(t)$, is monotonically increasing, which is assumed to be a linear path, expressed as $X(t)=\varphi+\beta t$, where $\varphi$ is the initial degradation level and $\beta$ is degradation rate. This linear path function can be applied to a wide range of degradation models.

Hard failure can be caused by fatal shocks that have a magnitude larger than $D_{j}(j=1,2,3$ ...). Nonfatal shocks impact the systems in three different ways: sudden increment in the degradation level at each shock $z, Y_{z}$, and the simultaneous transitions in the hard failure threshold $D_{j}$ and the degradation rate $\beta_{j}$, whenever the condition of the generalized mixed shock model is satisfied. As shown in Figure 2, the first transition in the hard failure threshold and the degradation rate occurs when the magnitude of the shock arrived at time $t_{2}$ is greater than the critical level for the extreme shock model $\left(W_{2}>D_{e}\right)$, which results in the reduced hard failure threshold from $D_{1}$ to $D_{2}$, and the increased degradation rate from $\beta_{1}$ to $\beta_{2}$. The second transition occurs when the time lag between the $3^{\text {rd }}$ and $4^{\text {th }}$ shocks is less than $\delta$, leading to the reduced hard failure threshold from $D_{2}$ to $D_{3}$, and the increased degradation rate from $\beta_{2}$ to $\beta_{3}$. 


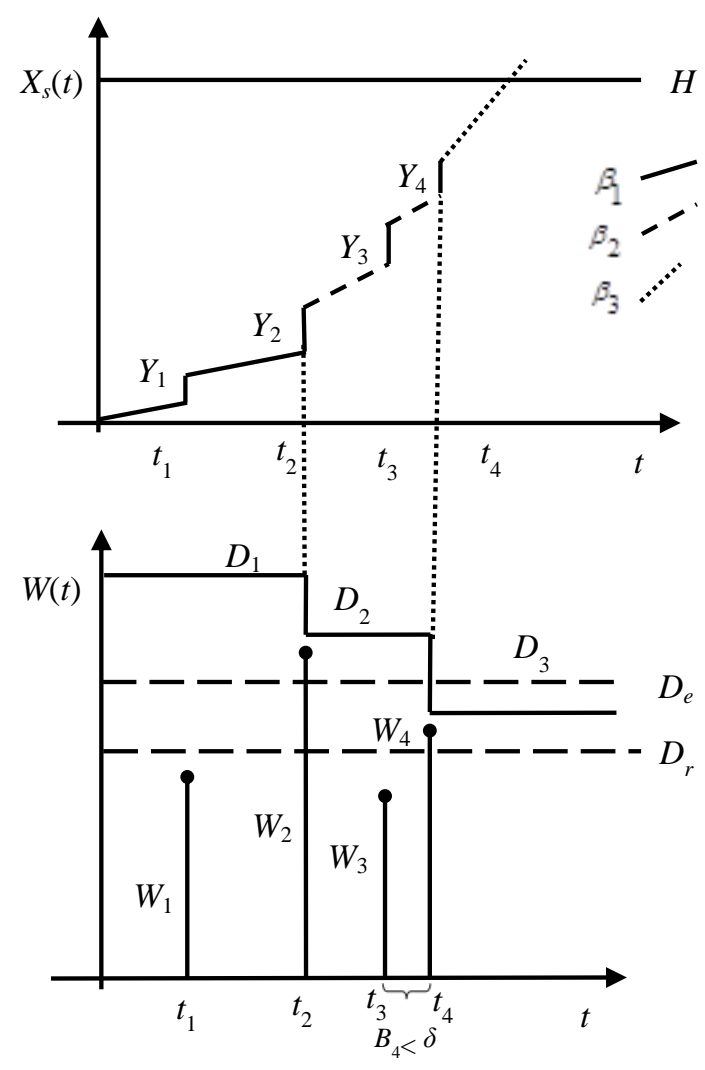

Figure 2: Two dependent competing failure processes: (a) soft failure, (b) hard failure

\section{Notations}

$D_{e} \quad$ Critical level for extreme shock model

$D_{r} \quad$ Critical level for run shock model, and $D_{e}>D_{r}$

$D_{j} \quad$ Hard failure threshold after the $j-1^{\text {th }}$ transition, $j=1,2, \ldots$

$\theta_{j} \quad$ Amount of reduction in the hard failure threshold after the $j-1^{\text {th }}$ transition, $j=1$, $2, \ldots$

$N(t) \quad$ Number of shocks arrived by time $t$

$\lambda \quad$ Arrival rate of shocks

$W_{z} \quad$ Magnitude of the shock load

$F_{W}(w) \quad$ Cumulative distribution function (cdf) of $W$

$H \quad$ Threshold level for soft failure

$X(t) \quad$ Amount of continuous degradation at time $t$

$X_{S}(t) \quad$ Degradation due to both continuous degradation and abrupt damage at time $t$

$\varphi \quad$ Initial level of degradation

$\beta_{j} \quad$ Degradation rate after the $j-1^{\text {th }}$ transition

$\eta_{j} \quad$ Amount of increase in the degradation rate after the $j-1^{\text {th }}$ transition, $j=1,2, \ldots$

$Y_{z} \quad$ Damage size on degradation caused by the shocks

$Z(t) \quad$ Cumulative damage size by shocks at time $t$ 
$n$

$B_{z} \quad$ Inter-arrival time between two consecutive shocks

$\delta$

$T_{j}$

$L$

$R(t)$

$R_{H}(t)$

$R_{S}(t)$

$S_{j}$

$S_{j}^{e}$

$S_{j}^{r}$

$S_{j}^{\delta}$

$I_{j+1}$ model

The $j^{\text {th }}$ transition time

Maximum number of possible transitions for a system

Reliability function by time $t$

Probability of no hard failure by time $t$

Probability of no soft failure by time $t$

Shock count that triggers the $j^{\text {th }}$ transition

Required number of consecutive shocks that are greater than $D_{r}$ in the run shock

Minimum time lag between two consecutive shocks for the $\delta$-shock model

Shock count that triggers the $j^{\text {th }}$ transition according to extreme shock model

Shock count that triggers the $j^{\text {th }}$ transition according to run shock model

Shock count that triggers the $j^{\text {th }}$ transition according to $\delta$-shock model

Number of remaining shocks after the $j^{\text {th }}$ transition, for $j=1, \ldots, l$

\subsection{Transition Process}

According to the proposed generalized mixed shock model, the degradation rate and the hard failure threshold shift simultaneously, if 1) a shock is above a critical value $D_{e}$ in the extreme shock model, 2) a time lag between two sequential shocks is less than $\delta$ in the $\delta$-shock model, or 3) a run of $n$ consecutive shocks are greater than a critical level $D_{r}\left(D_{r}<D_{e}\right)$ in the run shock model. These three classic shock models are competing against each other, and whichever occurs first causes the shift/transition in the hard failure threshold and the degradation rate. Some important assumptions regarding the transitions include:

1. If more than one shock pattern takes place at the same time, they do not amplify the effects on the hard failure threshold and the degradation rate.

2. The transition in the hard failure threshold and the degradation rate is not a onetime event, and can happen multiple times when the condition for the generalized mixed shock model is satisfied.

3. In practice, every time a transition occurs, the condition of the system worsens. Therefore, the system can only undergo a limited number of transitions, after which any transition can 
cause the system to fail. The maximum number of possible transitions for a system, $L$, is predetermined based on the device characteristics, such as the amounts of changes in the hard failure threshold/degradation rate after each transition.

The degradation rate increases from $\beta_{j}$ to $\beta_{j+1}$ when the system experiences the $j^{\text {th }}$ transition for $j=1, \ldots, l$, where $l$ is the total number of transitions actually occurred before failure, and $l \leq L$. The $\boldsymbol{\beta}$ representing the degradation rate vector is

$$
\boldsymbol{\beta}=\left[\begin{array}{lllll}
\beta_{1} & \cdots & \beta_{j} & \cdots & \beta_{l+1}
\end{array}\right]
$$

where the $j^{\text {th }}$ element $\left(\beta_{j}\right)$ is the degradation rate after the $(j-1)^{\text {th }}$ transition. We define $\beta_{j+1}=\beta_{j}+\eta_{j}$ where $\eta_{j}$ is an independent and identically distributed (i.i.d.) positive random variable and $\eta_{1}$ is independent of $\beta_{1}$.

Similarly, the hard failure threshold declines from $D_{j}$ to $D_{j+1}$ after the $j^{\text {th }}$ transition for $j=$ $1, \ldots, l$. We use $\mathbf{D}$ to represent the hard failure threshold vector as

$$
\mathbf{D}=\left[\begin{array}{lllll}
D_{1} & \cdots & D_{j} & \cdots & D_{l+1}
\end{array}\right]
$$

where the $j^{\text {th }}$ element $\left(D_{j}\right)$ is the hard failure threshold after the $(j-1)^{\text {th }}$ transition. We define $D_{j+1}$ $=D_{j}-\theta_{j}$ where $\theta_{j}$ is an i.i.d. positive random variable and $\theta_{1}$ is independent of $D_{1}$.

The shock that triggers a transition in the hard failure threshold and degradation rate by satisfying the condition for the generalized mixed shock model is called the trigger shock. The time lag between two successive transitions is defined as the transition time. To facilitate the evaluation of reliability for a system subject to changing hard failure threshold and changing degradation rate according to multiple competing shock patterns, we investigate each transition separately from others. Consequently, the trigger shock count (the number of shocks leading to a transition) and the transition time are being reset after each transition. In addition, after each transition, prior shocks are not taken into account for the next generalized mixed shock model. 
The number of shocks arrived after the $(j-1)^{\text {th }}$ transition that leads to the $j^{\text {th }}$ transition is denoted as $S_{j}$, which is an unknown random variable. The time lag between the $(j-1)^{\text {th }}$ and the $j^{\text {th }}$ trigger shocks is called the $j^{\text {th }}$ transition time, $T_{j}$, which is also a random variable. We use $\mathbf{T}$ to denote the transition time vector including $T_{j}$ for $j=1, \ldots, l$ :

$$
\mathbf{T}=\left[\begin{array}{llllll}
T_{1} & \cdots & T_{j} & \cdots & T_{l} & T_{l+1}
\end{array}\right]
$$

where $T_{l+1}=t-\sum_{j=1}^{l} T_{j}$ represents the remaining time after the last transition. After the random transition times are realized, the vector $\mathbf{T}$ transfers to be a vector composed of known values of transition times:

$$
\tilde{\mathbf{t}}=\left[\begin{array}{llllll}
t_{1} & \cdots & t_{j} & \cdots & t_{l} & t_{l+1}
\end{array}\right]
$$

\subsection{Shock Process and Hard Failure}

Random shocks are assumed to arrive according to a homogeneous Poisson process with rate $\lambda$, and it is independent of the degradation process and the shock magnitude. The total number of shocks by time $t, N(t)$, follows a Poisson distribution:

$$
P(N(t)=i)=\frac{e^{-\lambda t}(\lambda t)^{i}}{i !}, \quad i=0,1,2, \ldots
$$

The magnitude of a shock is denoted by $W_{z}$ for $z=1,2, \ldots$, a sequence of i.i.d. nonnegative random variables with a common cumulative distribution $F_{W}(w)=P\left(W_{z}<w\right)$, and $W_{z}$ is independent from the hard failure threshold $D_{j}$. In this paper, the traditional extreme shock model is employed for traumatic failure. It means that the unit fails as soon as the size of any shock is greater than the corresponding hard failure threshold at the time of shock arrival. The probability that a unit survives after exposing to a single shock is

$$
P\left(W_{z}<D_{j}\right)=F_{W}\left(D_{j}\right), \quad \text { for } z=1,2, \ldots ; j=1, \ldots, \text { or } l+1
$$


In order for a unit not to experience hard failure by time $t$, it needs to survive all the shocks by that time. By considering the following two scenarios, the probability of no hard failure by time $t, R_{H}(t)$, can be derived further.

i) No transition in the hard failure threshold occurs by time $t$, or none of shocks arrived by time $t$ meets the condition for the generalized mixed shock model to be able to damage the system. Therefore, the shock count for the first transition is larger than $N(t), S_{1}>N(t)=i$. Since no transition takes place, the hard failure threshold stays at its initial level of $D_{1}$ and the magnitude of each shock must be less than $D_{1}$. The probability of surviving against hard failure given that no transition occurs by time $t$ is

$$
R_{H}\left(t \mid S_{1}>N(t)=i\right)=P\left(\bigcap_{z=1}^{i}\left\{W_{z}<D_{1}\right\}\right)=F_{W}\left(D_{1}\right)^{i}
$$

ii) At least one transition occurs by time $t$, or at least one shock arrives by time $t$ that meets the condition for the generalized mixed shock model to damage the system. Therefore, the shock count for the first transition is less than or equal to $N(t), S_{1} \leq N(t)=i$. The magnitude of each shock must be less than the corresponding hard failure threshold at the time of shock arrival.

We use $I_{j+1}=i-\sum_{k=1}^{j} S_{k}$, for $j=1, \ldots, l$, to denote the number of remaining shocks after the $j^{\text {th }}$ transition, which is a random variable. After the values for shock counts are realized, it becomes $i_{j+1}=i-\sum_{k=1}^{j} s_{k}$. The probability of no hard failure given $S_{1} \leq N(t)=i$ is (see Appendix A)

$$
R_{H}\left(t \mid S_{1}=s_{1} \leq N(t)=i\right)=\sum_{s_{l}=1}^{i_{l}} \cdots \sum_{s_{2}=1}^{i_{2}} \sum_{l=1}^{L}\left[\prod_{j=1}^{l} F_{W}\left(D_{j}\right)^{s_{j}}\right] F_{W}\left(D_{l+1}\right)^{i_{l+1}} P\left(S_{l+1}>i_{l+1}\right)\left[\prod_{j=2}^{l} P\left(S_{j}=s_{j}\right)\right]
$$

Since the hard failure threshold is reduced to a lower level after a transition, Equation (4) ensures that the magnitude of each shock of $s_{j}$ shocks arrived between $(j-1)^{\text {th }}$ and $j^{\text {th }}$ transitions is 
less than the corresponding hard failure threshold at the time of shock arrival, $D_{j}$, while $s_{j}$ can vary from 1 to $i_{j}$ for $j=1, \ldots, l$ and $l=1, \ldots, L$. We present the further derivation of the last two terms in Equation (4) in Section 4, where $P\left(S_{l+1}>i_{l+1}\right)$ is the probability that no transition occurs after the very last transition $l$, and $P\left(S_{j}=s_{j}\right)$ is the probability that the $s_{j}^{\text {th }}$ shock after the $(j-1)^{\text {th }}$ transition meets the condition for the generalized mixed shock model leading to the $j^{\text {th }}$ transition.

\subsection{Degradation Process and Soft Failure}

A soft failure occurs when the overall degradation exceeds a predetermined threshold $H$. As aforementioned, we model the degradation process as a linear path, expressed as $X(t)=\varphi+\beta t$, where $\varphi$ is the initial degradation level that is a random variable (due to variability in manufacturing and delivering processes) and $\beta$ is the degradation rate that is a random variable and varies from part to part.

The shock process impacts the degradation in two ways: causing a sudden increment to the degradation by each nonfatal shock, and increasing the degradation rate according to a generalized mixed shock model. We denote the damage from each shock by $Y_{z}$ for $z=1,2 \ldots$, where $Y_{z}$ is a sequence of i.i.d. non-negative random variables. The overall degradation including both continuous degradation and instantaneous damage induced by nonfatal shocks can be expressed as

$$
X_{S}(t)=X(t)+Z(t)=X(t)+\sum_{z=1}^{N(t)} Y_{z},
$$

where $Z(t)$ represents the cumulative damage by nonfatal random shocks, and $Y_{z}$ is independent from the continuous degradation $X(t)$.

By considering the following two situations, the probability of no soft failure by time $t$, $R_{S}(t)$, can be derived further. 
i) No transition in both the hard failure threshold and the degradation rate occurs by time $t$, or the condition for the generalized mixed shock model is not met by any shock by time $t$. Therefore, the shock count for the first transition is larger than $N(t), S_{1}>N(t)=i$. Given no transition by time, the initial degradation rate is fixed and the probability of no soft failure by time $t$ is

$$
R_{S}\left(t \mid S_{1}>N(t)=i\right)=P\left(X_{S}(t)<H \mid S_{1}>N(t)=i\right)=P\left(\varphi+\beta_{1} t+\sum_{z=1}^{i} Y_{z}<H\right) .
$$

ii) There is at least one shock by time $t$ that meets the condition of the generalized mixed shock model to accelerate the degradation rate. Therefore, the shock count for that trigger shock is less than or equal to $N(t), S_{1} \leq N(t)=i$. When a system experiences a transition, the degradation rate shifts; therefore, the degradation progresses at an increased degradation rate. Similar to Equation (4), the probability of no soft failure given at least one degradation rate transition is derived as follows (see Appendix B)

$$
\begin{aligned}
R_{S}\left(t \mid S_{1}=s_{1} \leq N(t)=i\right) & =\sum_{s_{l}=1}^{i_{l}} \cdots \sum_{s_{2}=1}^{i_{2}} \sum_{l=1}^{L} P\left(\varphi+\sum_{j=1}^{l} \beta_{j} T_{j}+\beta_{l+1} T_{l+1}+\sum_{z=1}^{i} Y_{z}<H\right) \\
& \times P\left(S_{l+1}>i_{l+1}\right)\left[\prod_{j=2}^{l} P\left(S_{j}=s_{j}\right)\right] .
\end{aligned}
$$

Since shocks arrive according to a homogeneous Poisson process, the arrival time for the $S_{j}$ shock (the trigger shock for the $j^{\text {th }}$ transition), $T_{j}$, given a value of $S_{j}=s_{j}$, follows a gamma distribution with the scale parameter of $s_{j}$ and the shape parameter of $\lambda$. Therefore the probability density function for the $j^{\text {th }}$ transition time given $S_{j}=s_{j}$ is

$$
f_{T_{j}}\left(t_{j}\right)=\frac{\lambda^{s_{j}}}{\left(s_{j}-1\right) !} t_{j}^{s_{j}-1} e^{-\lambda t_{j}}, \text { for } j=1, \ldots, l .
$$

Let $t_{j}^{\prime}=t-\sum_{k=1}^{j-1} t_{k}$ denote the remaining time after the $j^{\text {th }}$ transition for $j=1, \ldots, l$. Then the conditional probability in (7) can be calculated as 


$$
\begin{aligned}
R_{S}\left(t \mid S_{1}=s_{1} \leq N(t)=i\right) & =\sum_{s_{l}=1}^{i_{l}} \cdots \sum_{s_{2}=1}^{i_{2}} \sum_{l=1}^{L} P\left(S_{l+1}>i_{l+1}\right)\left[\prod_{j=2}^{l} P\left(S_{j}=s_{j}\right)\right] \\
& \times\left(\int_{0}^{t} \cdots\left(\int_{0}^{t_{l}^{\prime}} P\left(\varphi+\sum_{j=1}^{l} \beta_{j} t_{j}+\beta_{l+1} t_{l+1}+\sum_{z=1}^{i} Y_{z}<H\right) f_{T_{l}}\left(t_{l}\right) d t_{l}\right) \cdots f_{T_{1}}\left(t_{1}\right) d t_{1}\right) .
\end{aligned}
$$

\subsection{System Reliability Modeling}

We investigate the reliability model for a system subject to the generalized mixed shock model that can damage the system by lowering the hard failure threshold and accelerating the degradation rate multiple times. The reliability for such a system is the probability that no hard failure and no soft failure occur by time $t$. We consider two situations: (1) no transition by time $t$, i.e., the trigger shock count is greater than the number of shocks arrived by time $t$, and (2) one or more transitions by time $t$, i.e., the shock count for the first transition is equal to or less than the number of shocks by time $t$. Accordingly, the reliability at time $t$ is derived based on Eqs. (3), (4), (6) and (9):

$$
\begin{aligned}
R(t)= & \left(R_{S}\left(t \mid S_{1}>N(t)\right) \cap R_{H}\left(t \mid S_{1}>N(t)\right)\right) P\left(S_{1}>N(t)\right) \\
& +\sum_{s_{1}=1}^{N(t)}\left(R_{S}\left(t \mid S_{1}=s_{1} \leq N(t)\right) \cap R_{H}\left(t \mid S_{1}=s_{1} \leq N(t)\right)\right) P\left(S_{1}=s_{1} \leq N(t)\right) \\
= & \sum_{i=0}^{\infty} R_{S}\left(t \mid S_{1}>N(t)=i\right) R_{H}\left(t \mid S_{1}>N(t)=i\right) P\left(S_{1}>N(t) \mid N(t)=i\right) P(N(t)=i) \\
& +\sum_{i=1}^{\infty} \sum_{s_{1}=1}^{N(t)} R_{S}\left(t \mid S_{1}=s_{1} \leq N(t)=i\right) R_{H}\left(t \mid S_{1}=s_{1} \leq N(t)=i\right) P\left(S_{1}=s_{1} \mid N(t)=i\right) P(N(t)=i) \\
= & P\left(\varphi+\beta_{1} t<H\right) \times e^{-\lambda t}+\sum_{i=1}^{\infty} P\left(\varphi+\beta_{1} t+\sum_{z=1}^{i} Y_{z}<H\right) F_{W}\left(D_{1}\right)^{i} P\left(S_{1}>i\right) \frac{e^{-\lambda t}(\lambda t)^{i}}{i !} \\
& +\sum_{i=1}^{\infty} \sum_{s_{l}=1}^{i_{l}} \ldots \sum_{s_{2}=1}^{i_{2}} \sum_{s_{1}=1}^{i} \sum_{l=1}^{L}\left(\int_{0}^{t} \ldots\left(\int_{0}^{t_{t}^{\prime}} P\left(\varphi+\sum_{j=1}^{l} \beta_{j} t_{j}+\beta_{l+1} t_{l+1}+\sum_{z=1}^{i} Y_{z}<H\right) f_{T_{l}}\left(t_{l}\right) d t_{l}\right) \cdots f_{T_{1}}\left(t_{1}\right) d t_{1}\right) \\
& \times\left[\prod_{j=1}^{l} F_{W}\left(D_{j}\right)^{s_{j}}\right] F_{W}\left(D_{l+1}\right)^{i_{l+1}} P\left(S_{l+1}>i_{l+1}\right)\left[\prod_{j=1}^{l} P\left(S_{j}=s_{j}\right)\right] \frac{e^{-\lambda t}(\lambda t)^{i}}{i !} .
\end{aligned}
$$


Given the number of shocks by time $t$, the soft failure and hard failure processes are conditionally independent, due to the assumption that the shock damage size $Y_{z}$ is independent of the shock load $W_{z}$. Therefore, Equation (10) is further derived as

$$
\begin{aligned}
R(t)= & \sum_{i=0}^{\infty} R_{S}\left(t \mid S_{1}>N(t)=i\right) R_{H}\left(t \mid S_{1}>N(t)=i\right) P\left(S_{1}>N(t) \mid N(t)=i\right) P(N(t)=i) \\
& +\sum_{i=1}^{\infty} \sum_{s_{1}=1}^{N(t)} R_{S}\left(t \mid S_{1}=s_{1} \leq N(t)=i\right) R_{H}\left(t \mid S_{1}=s_{1} \leq N(t)=i\right) P\left(S_{1}=s_{1} \mid N(t)=i\right) P(N(t)=i) \\
= & P\left(\varphi+\beta_{1} t<H\right) \times e^{-\lambda t}+\sum_{i=1}^{\infty} P\left(\varphi+\beta_{1} t+\sum_{z=1}^{i} Y_{z}<H\right) F_{W}\left(D_{1}\right)^{i} P\left(S_{1}>i\right) \frac{e^{-\lambda t}(\lambda t)^{i}}{i !} \\
& +\sum_{i=1}^{\infty} \sum_{s_{l}=1}^{i_{l}} \ldots \sum_{s_{2}=1}^{i_{2}} \sum_{s_{1}=1}^{i} \sum_{l=1}^{L}\left(\int_{0}^{t} \cdots\left(\int_{0}^{t_{l}^{\prime}} P\left(\varphi+\sum_{j=1}^{l} \beta_{j} t_{j}+\beta_{l+1} t_{l+1}+\sum_{z=1}^{i} Y_{z}<H\right) f_{T_{l}}\left(t_{l}\right) d t_{l}\right) \cdots f_{T_{1}}\left(t_{1}\right) d t_{1}\right) \\
& \times\left[\prod_{j=1}^{l} F_{W}\left(D_{j}\right)^{s_{j}}\right] F_{W}\left(D_{l+1}\right)^{i_{l+1}} P\left(S_{l+1}>i_{l+1}\right)\left[\prod_{j=1}^{l} P\left(S_{j}=s_{j}\right)\right] \frac{e^{-\lambda t}(\lambda t)^{i}}{i !} .
\end{aligned}
$$

The general reliability model can be reduced to special cases when relaxing different assumptions. The result for a special case when the maximum number of transitions allowed $(L)$ is equal to 1 (anymore transition can cause the system to fail) is presented in Appendix C.

\section{Probability of Trigger Shock Count in the Generalized Mixed Shock Model}

The probabilities related to $S_{j}$ (the trigger shock count for the $j^{\text {th }}$ transition) in (10) are derived in the following sections.

\subsection{Applicability of Different Shock Models}

A transition occurs when the condition is satisfied for at least one of three shock patterns in the generalized mixed shock model: extreme shock model, $\delta$-shock model, and run shock model. It is likely that the conditions for two or more shock patterns are satisfied at the same shock. For example, a shock with a size greater than $D_{e}$ (extreme shock pattern) can have a time lag that is shorter than $\delta$ ( $\delta$-shock pattern). In a transition, the hard failure threshold is assumed to 
shift to a lower level. If the resulting hard failure threshold $D_{j}$ is less than $D_{e}$ in the extreme shock model or $D_{r}$ in the run shock model, the corresponding shock pattern is not applicable in the generalized mixed shock model anymore. For example, when $D_{j}$ is less than $D_{e}$, a shock larger than $D_{j}$ leads to a hard failure even before it is considered in the extreme shock model for triggering a transition.

Depending on the applicability of different shock patterns, we derive the probability of the $j^{\text {th }}$ transition occurring at the $s_{j}^{\text {th }}$ shock, $P\left(S_{j}=s_{j}\right)$, in the following. Due to the independence between the shock arrival time and the shock magnitude, the occurrence of $\delta$-shock model is probabilistically independent from the occurrence of extreme shock model and run shock model.

1. At any time, if the hard failure threshold is greater than the critical level of extreme shock model $\left(D_{j}>D_{e}>D_{r}\right)$, all three shock patterns are applicable. Then the probability of the $j^{\text {th }}$ transition at the $s_{j}^{\text {th }}$ shock equals to the probability that any of three shock patterns may cause that transition:

$$
\begin{aligned}
P\left(S_{j}=s_{j}\right)= & P\left(S_{j}^{e}=s_{j} \cup S_{j}^{r}=s_{j} \cup S_{j}^{\delta}=s_{j}\right) \\
= & P\left(S_{j}^{e}=s_{j}\right)+P\left(S_{j}^{r}=s_{j}\right)+P\left(S_{j}^{\delta}=s_{j}\right) \\
& -P\left(S_{j}^{r}=s_{j} \mid S_{j}^{e}=s_{j}\right) P\left(S_{j}^{e}=s_{j}\right)-P\left(S_{j}^{e}=s_{j}\right) P\left(S_{j}^{\delta}=s_{j}\right) \\
& -P\left(S_{j}^{r}=s_{j}\right) P\left(S_{j}^{\delta}=s_{j}\right)+P\left(S_{j}^{r}=s_{j} \mid S_{j}^{e}=s_{j}\right) P\left(S_{j}^{e}=s_{j}\right) P\left(S_{j}^{\delta}=s_{j}\right) .
\end{aligned}
$$

2. If the hard failure threshold is between the critical levels of extreme shock model and run shock model $\left(D_{e}>D_{j}>D_{r}\right)$, the extreme shock model is not applicable. Then $P\left(S_{j}=s_{j}\right)$ equals to the probability of run shock model or $\delta$-shock model that causes the $j^{\text {th }}$ transition:

$$
\begin{aligned}
P\left(S_{j}=s_{j}\right) & =P\left(S_{j}^{r}=s_{j} \cup S_{j}^{\delta}=s_{j}\right) \\
& =P\left(S_{j}^{r}=s_{j}\right)+P\left(S_{j}^{\delta}=s_{j}\right)-P\left(S_{j}^{r}=s_{j}\right) P\left(S_{j}^{\delta}=s_{j}\right) .
\end{aligned}
$$


3. If the hard failure threshold is less than the critical level of run shock model $\left(D_{r}>D_{j}\right)$, only $\delta$-shock model could be the reason for a transition:

$$
P\left(S_{j}=s_{j}\right)=P\left(S_{j}^{\delta}=s_{j}\right)
$$

\subsection{Probability of Trigger Shock Count}

The probabilities for different shock patterns occurring at the $s_{j}^{\text {th }}$ shock in Eqs. (11-13) are derived and presented in Table 1. The first column lists different shock patterns that can cause the $j^{\text {th }}$ transition, and the second column presents the probabilities of the corresponding shock patterns.

Table 1: Probability for occurrence of different shock patterns

\begin{tabular}{|c|c|c|}
\hline \multicolumn{2}{|c|}{$\begin{array}{c}\begin{array}{c}\text { Shock models that } \\
\text { cause the } j^{\text {th }} \text { transition } \\
\text { at the } s_{j}^{\text {th }} \text { shock }\end{array} \\
\end{array}$} & Probability \\
\hline \multicolumn{2}{|c|}{$\begin{array}{l}\text { 1) Extreme shock pattern } \\
\text { at the } s_{j}^{\text {th }} \text { shock }\end{array}$} & $P\left(S_{j}^{e}=s_{j}\right)=P\left(\bigcap_{z=1}^{s_{j}-1}\left\{W_{z}<D_{e}\right\}, W_{s_{j}} \geq D_{e} \mid \bigcap_{z=1}^{s_{j}}\left\{W_{z}<D_{j}\right\}\right)=\frac{F_{W}\left(D_{e}\right)^{s_{j}-1}\left(F_{W}\left(D_{j}\right)-F_{W}\left(D_{e}\right)\right)}{F_{W}\left(D_{j}\right)^{s_{j}}}$ \\
\hline \multicolumn{2}{|c|}{$\begin{array}{l}\text { 2) } \delta \text {-shock pattern at the } \\
s_{j}^{\text {th }} \text { shock }\end{array}$} & $P\left(S_{j}^{\delta}=s_{j}\right)=P\left(\bigcap_{z=1}^{s_{j}-1}\left\{B_{z}>\delta\right\}, B_{s_{j}} \leq \delta\right)=e^{-\left(s_{j}-1\right) \lambda \delta}\left(1-e^{-\lambda \delta}\right)$ \\
\hline \multirow{3}{*}{$\begin{array}{l}\text { 3) Run shock } \\
\text { pattern at the } \\
s_{j}^{\text {th }} \text { shock }\end{array}$} & $s_{j}<n$ & $P\left(S_{j}^{r}=s_{j}\right)=0$ \\
\hline & $s_{j}=n$ & $P\left(S_{j}^{r}=s_{j}\right)=P\left(\bigcap_{z=1}^{n}\left\{W_{z} \geq D_{r}\right\} \mid \bigcap_{z=1}^{n}\left\{W_{z}<D_{j}\right\}\right)=\frac{\left(F_{W}\left(D_{j}\right)-F_{W}\left(D_{r}\right)\right)^{n}}{F_{W}\left(D_{j}\right)^{n}}$ \\
\hline & $s_{j}>n$ & $\begin{aligned} P\left(S_{j}^{r}=s_{j}\right) & =P\left(S_{j}^{r}>s_{j}-n-1\right) P\left(W_{s_{j}-n}<D_{r}, \bigcap_{z=s_{j}-n+1}^{s_{j}}\left\{W_{z} \geq D_{r}\right\} \mid \bigcap_{z=s_{j}-n}^{s_{j}}\left\{W_{z}<D_{j}\right\}\right. \\
& =P\left(S_{j}^{r}>s_{j}-n-1\right) \frac{F_{W}\left(D_{r}\right)\left(F_{W}\left(D_{j}\right)-F_{W}\left(D_{r}\right)\right)^{n}}{F_{W}\left(D_{j}\right)^{n+1}}\end{aligned}$ \\
\hline \multirow{2}{*}{$\begin{array}{l}\text { 4) Run shock } \\
\text { pattern at the } \\
s_{j}^{\text {th }} \text { shock } \\
\text { given } \\
\text { concurrent } \\
\end{array}$} & $s_{j}<n$ & $P\left(S_{j}^{r}=s_{j} \mid S_{j}^{e}=s_{j}\right)=0$ \\
\hline & $s_{j}=n$ & $P\left(S_{j}^{r}=s_{j} \mid S_{j}^{e}=s_{j}\right)=P\left(\bigcap_{z=1}^{n}\left\{D_{r} \leq W_{z}\right\} \mid \bigcap_{z=1}^{n-1}\left\{W_{z}<D_{e}\right\}, W_{n} \geq D_{e}\right)=\frac{\left(F_{W}\left(D_{e}\right)-F_{W}\left(D_{r}\right)\right)^{n-1}}{F_{W}\left(D_{e}\right)^{n-1}}$ \\
\hline
\end{tabular}




\begin{tabular}{|l|r|r|}
\hline $\begin{array}{l}\text { extreme } \\
\text { shock pattern }\end{array}$ & $P\left(S_{j}^{r}=s_{j} \mid S_{j}^{e}=s_{j}\right)=$ & $P\left(S_{j}^{r}>s_{j}-n-1 \mid \bigcap_{z=1}^{s_{j}-n-1}\left\{W_{z}<D_{e}\right\}\right)$ \\
& & $\times P\left(W_{s_{j}-n}<D_{r}, \bigcap_{z=s_{j}-n+1}^{s_{j}}\left\{D_{r} \leq W_{z}\right\} \mid \bigcap_{z=s_{j}-n}^{s_{j}-1}\left\{W_{z}<D_{e}\right\}, W_{s_{j}} \geq D_{e}\right)$ \\
& $=P\left(S_{j}^{r}>s_{j}-n-1 \mid \bigcap_{z=1}^{s_{j}-n-1}\left\{W_{z}<D_{e}\right\}\right) \frac{F_{W}\left(D_{r}\right)\left(F_{W}\left(D_{e}\right)-F_{W}\left(D_{r}\right)\right)^{n-1}}{F_{W}\left(D_{e}\right)^{n}}$ \\
&
\end{tabular}

When the run shock model is involved, we have three subcases based on the relationship between $s_{j}$ and $n$, as presented in Table 1 . In general, we defined $V_{s j}$ to be the probability that no run of $n$ consecutive successes (a success is defined as a shock greater than $D_{r}$ ) occur in a set of $s_{j}$ shocks, and $V_{s j}^{\prime}$ to be the probability that the first run of $n$ consecutive successes occurs at the $s_{j}^{\text {th }}$ shock. We have (Krieger 1984):

$$
V_{s_{j}}=\left\{\begin{array}{lr}
1, & \text { if } 0 \leq s_{j}<n \\
1-p^{n}, & \text { if } s_{j}=n, \\
q V_{s_{j}-1}+\ldots+q p^{n-1} V_{s_{j}-n}, & \text { if } s_{j}>n
\end{array}\right.
$$

where $p$ is the probability of success, and $q=1-p$. In addition, we have

$$
V_{s_{j}}^{\prime}= \begin{cases}0, & \text { if } \quad 0 \leq s_{j}<n \\ p^{n}, & \text { if } s_{j}=n . \\ q p^{n} V_{s_{j}-n-1}, & \text { if } s_{j}>n\end{cases}
$$

When $s_{j}>n$, to have the first run of $n$ consecutive successes occurring at the $s_{j}^{\text {th }}$ trial, the last $n$ trials must be successes, the $\left(s_{j}-n\right)^{\text {th }}$ trial must be a failure, and no consecutive $n$ successes occur in the first $s_{j}-n-1$ trials.

The derivation of the four cases in Table 1 is described as follows:

1) Extreme shock pattern: the probability is derived for the situation when the $s_{j}^{\text {th }}$ shock is the first shock with a magnitude greater than $D_{e}$, and the prior $s_{j}-1$ shocks are less than $D_{e}$, given that the magnitudes of all shocks are less than $D_{j}$ to ensure no occurrence of hard failure. 
2) $\delta$-shock pattern: the probability is derived for the situation when the time lag between the $s_{j}-1^{\text {th }}$ shock and the $s_{j}^{\text {th }}$ shock is shorter than $\delta$, while the prior $s_{j}-1$ time lags are longer than $\delta$.

3) Run shock pattern: the probability is derived based on the relationship between $s_{j}$ and $n$. Eq. (15) is used in three different subcases to derive the probability of the first run of $n$ consecutive shocks greater than $D_{r}$ occurs at the $s_{j}^{\text {th }}$ shock, given that the magnitude for all $s_{j}$ shocks is less than $D_{j}$ (to ensure no hard failure).

4) Run shock pattern given concurrent occurrence of extreme shock pattern at the $s_{j}^{\text {th }}$ shock: according to Eq. (15), when the run shock model is among shock patterns that can cause a transition, we have three subcases based on the relationship between $s_{j}$ and $n$. The probability is derived considering that the first run of $n$ consecutive shocks greater than $D_{r}$ occurs at the $s_{j}^{\text {th }}$ shock, given the first $s_{j}-1$ shock magnitudes are less than $D_{e}$ and the $s_{j}^{\text {th }}$ shock magnitude is greater than $D_{e}$.

\subsection{Probability of No Transition in Remaining Shocks}

We assume that the system undergoes a finite number of transitions $l, l=0, \ldots, L$. When the number of transitions in the degradation rate and the hard failure threshold reaches $l$, it implies that there is no transition in the remaining $i_{l+1}$ shocks after the $t^{\text {th }}$ transition. Therefore, none of the $i_{l+1}$ shocks occurred after the $l^{\text {th }}$ transition meets the condition for the generalized mixed shock model, i.e., the shock count for the $l+1$ transition is larger than $i_{l+1}$. The probability of no transition in the last $i_{l+1}$ shocks depends on the relationship between the final hard failure threshold $D_{l+1}$ after the $l^{\text {th }}$ transition and the critical levels for extreme shock pattern $\left(D_{e}\right)$ and run shock pattern $\left(D_{r}\right)$. 
1. If the hard failure threshold after the $l^{\text {th }}$ transition is greater than the critical level of extreme shock model $\left(D_{l+1}>D_{e}>D_{r}\right)$, all three shock patterns are applicable. Then the probability of no transition in the last $i_{l+1}$ shocks equals to the probability that none of these three shock patterns happens among the $i_{l+1}$ shocks.

$$
\begin{aligned}
P\left(S_{l+1}>i_{l+1}\right) & =P\left(S_{l+1}^{e}>i_{l+1} \cap S_{l+1}^{r}>i_{l+1} \cap S_{l+1}^{\delta}>i_{l+1}\right)= \\
& =P\left(S_{l+1}^{r}>i_{l+1} \mid S_{l+1}^{e}>i_{l+1}\right) P\left(S_{l+1}^{e}>i_{l+1}\right) P\left(S_{l+1}^{\delta}>i_{l+1}\right) .
\end{aligned}
$$

2. If the hard failure threshold after the $l^{\text {th }}$ transition is between the critical levels of extreme shock model and run shock model $\left(D_{e}>D_{l+1}>D_{r}\right)$, the extreme shock model is not applicable. Then we have

$$
P\left(S_{l+1}>i_{l+1}\right)=P\left(S_{l+1}^{r}>i_{l+1} \cap S_{l+1}^{\delta}>i_{l+1}\right)=P\left(S_{l+1}^{r}>i_{l+1}\right) P\left(S_{l+1}^{\delta}>i_{l+1}\right) .
$$

3. If the hard failure threshold after the $l^{\text {th }}$ transition is less than the critical level of run shock model $\left(D_{r}>D_{l+1}\right)$, only $\delta$-shock model can damage the system.

$$
P\left(S_{l+1}>i_{l+1}\right)=P\left(S_{l+1}^{\delta}>i_{l+1}\right) .
$$

The probabilities of no transition after the $l^{\text {th }}$ transition in the remaining $i_{l+1}$ shocks, or $P\left(S_{l+1}>i_{l+1}\right)$, for different scenarios in Eqs. (16-18) are derived and presented in Table 2. The derivation of the four cases in Table 2 is described as follows. As explained in Section 4.2, when the run shock model is applicable, there are three subcases based on the relationship between $i_{l+1}$ and $n$.

1) No extreme shock pattern: the probability is derived for the situation when the magnitudes of all remaining $i_{l+1}$ shocks after the last transition are less than $D_{e}$, given that the magnitudes of all shocks are less than $D_{l+1}$ to ensure no occurrence of hard failure.

2) No $\delta$-shock pattern: the probability is derived for the situation when the time lags between any two successive shocks among the remaining $i_{l+1}$ shocks must be longer than $\delta$. 
3) No run shock pattern: the probability of no transition due to the run shock pattern using Eq. (14) is derived for three different subcases based on the relationship between $i_{l+1}$ and $n$, given the magnitudes of all shocks are less than $D_{l+1}$.

4) No run shock pattern given no extreme shock pattern in the remaining $i_{l+1}$ shocks: Eq. (14) is used to further derive the probability of no set of $n$ consecutive shocks that are greater than $D_{r}$ (no run shock pattern), given that the magnitudes of all shocks are less than $D_{e}$ (no extreme shock pattern) for three subcases.

Table 2: Probability of no transition after the $l^{\text {th }}$ transition

\begin{tabular}{|c|c|c|}
\hline \multirow{2}{*}{\multicolumn{2}{|c|}{ 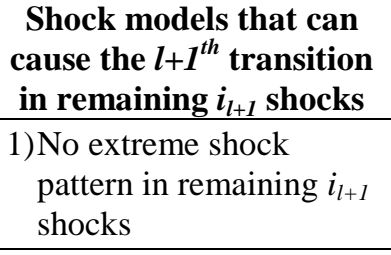 }} & Probability \\
\hline & & $P\left(S_{l+1}^{e}>i_{l+1}\right)=P\left(\bigcap_{z=1}^{i_{l+1}}\left\{W_{z}<D_{e}\right\} \bigcap_{z=1}^{i_{l+1}}\left\{W_{z}<D_{l+1}\right\}\right)=\frac{F_{W}\left(D_{e}\right)^{i_{l+1}}}{F_{W}\left(D_{l+1}\right)^{i_{l+1}}}$ \\
\hline \multicolumn{2}{|c|}{$\begin{array}{l}\text { 2) No } \delta \text {-shock pattern in } \\
\text { remaining } i_{l+1} \text { shocks }\end{array}$} & $P\left(S_{l+1}^{\delta}>i_{l+1}\right)=P\left(\bigcap_{z=1}^{i_{l+1}}\left\{B_{z}>\delta\right\}\right)=e^{-i_{l+1} \lambda \delta}$ \\
\hline \multirow[b]{3}{*}{$\begin{array}{l}\text { 3) No run shock } \\
\text { pattern in } \\
\text { remaining } i_{l+l} \\
\text { shocks }\end{array}$} & $i_{l+1}<n$ & $P\left(S_{l+1}^{r}>i_{l+1}\right)=1$ \\
\hline & $i_{l+1}=n$ & $P\left(S_{l+1}^{r}>i_{l+1}\right)=1-P\left(\bigcap_{z=1}^{i_{l+1}}\left\{W_{z} \geq D_{r}\right\} \mid \bigcap_{z=1}^{i_{l+1}}\left\{W_{z}<D_{l+1}\right\}\right)=1-\frac{\left(F_{W}\left(D_{l+1}\right)-F_{W}\left(D_{r}\right)\right)^{n}}{F_{W}\left(D_{l+1}\right)^{n}}$ \\
\hline & $i_{l+1}>n$ & $\begin{array}{l}P\left(S_{l+1}^{r}>i_{l+1}\right)=\left(S_{l+1}^{r}>i_{l+1}-1\right) P\left(W_{i_{l+1}}<D_{r} \mid W_{i_{l+1}}<D_{l+1}\right)+\cdots \\
\cdots+P\left(S_{l+1}^{r}>i_{l+1}-n\right) P\left(W_{i_{l+1}-n+1}<D_{r}, \bigcap_{z=i_{l+1}-n+2}^{i_{l+1}}\left\{W_{z} \geq D_{r}\right\} \mid \bigcap_{z=i_{l+1}-n+1}^{i_{l+1}}\left\{W_{z}<D_{l+1}\right\}\right) \\
=P\left(S_{l+1}^{r}>i_{l+1}-1\right) \frac{F_{W}\left(D_{r}\right)}{F_{W}\left(D_{l+1}\right)}+\cdots+P\left(S_{l+1}^{r}>i_{l+1}-n\right) \frac{F_{W}\left(D_{r}\right)\left(F_{W}\left(D_{l+1}\right)-F_{W}\left(D_{r}\right)\right)^{n-1}}{F_{W}\left(D_{l+1}\right)^{n}}\end{array}$ \\
\hline \multirow[b]{2}{*}{$\begin{array}{l}\text { 4) No run shock } \\
\text { pattern in } \\
\text { remaining } i_{l+1} \\
\text { shocks given no } \\
\text { extreme shock } \\
\text { pattern }\end{array}$} & $i_{l+1}<n$ & $P\left(S_{l+1}^{r}>i_{l+1}-1 \mid S_{l+1}^{e}>i_{l+1}-1\right)=1$ \\
\hline & $i_{l+1}=n$ & $\begin{aligned} P\left(S_{l+1}^{r}>i_{l+1}-1 \mid S_{l+1}^{e}>i_{l+1}-1\right) & =1-P\left(\bigcap_{z=1}^{n}\left\{D_{r} \leq W_{z}\right\} \mid \bigcap_{z=1}^{n}\left\{W_{z}<D_{e}\right\}\right) \\
& =1-\frac{\left(F_{W}\left(D_{e}\right)-F_{W}\left(D_{r}\right)\right)^{n}}{F_{W}\left(D_{e}\right)^{n}}\end{aligned}$ \\
\hline
\end{tabular}




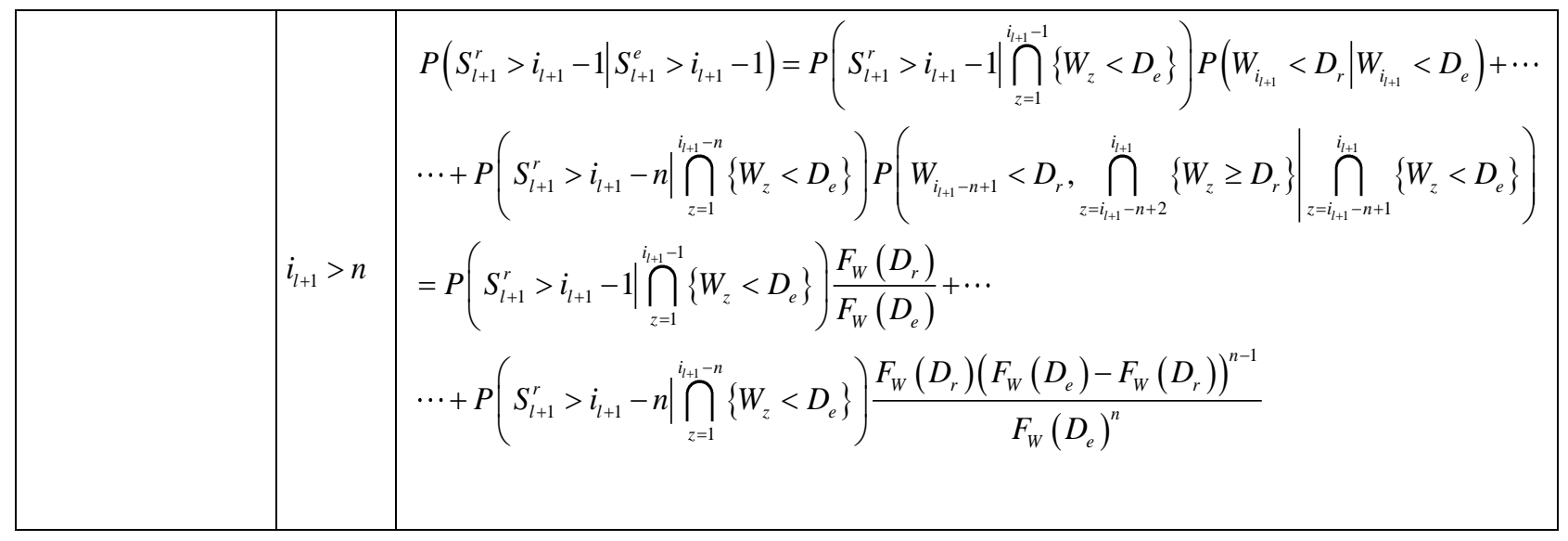

\section{Numerical Example}

The developed reliability model is illustrated by using a realistic example of MEMS. Emerged in the late 1980s, MEMS devices are composed of (a) micro mechanical-structures, (b) micro-sensors, (c) micro-electronics, and (d) micro-actuators, all placed into one device at microscale. They have been widely used in many applications such as medical, aerospace, military, etc. (Ye et al. 2012).

We implement our new reliability models to the application of micro-engines, because a micro-engine can fail due to two competing failure processes: (1) soft failure as the result of aging degradation and debris caused by shocks, and (2) hard failure because of spring fracture induced from the same shock process (Tanner et al. 2000). In addition, after exposure to a certain pattern of shocks or a significantly large shock, a micro-engine becomes more susceptible to shocks with a decreasing hard failure threshold, and the wear volume accumulates faster. Using data from Tanner \& Dugger (2003) and Peng et al. (2011), along with some reasonable assumptions, the values for parameters are presented in Table 3. Numerical integration in Matlab has been applied for computing the probabilities in the numerical example.

For the proposed generalized mixed shock model with increasing degradation rate and decreasing hard failure threshold, the reliability function $R(t)$ in (10) is plotted in Figure 3 , and 
sensitivity analysis is performed to measure the effects of the parameters $D_{e}, \delta$ and $D_{r}$ on the reliability function (Fig. 4-6).

Table 3: Parameter Values

\begin{tabular}{|c|c|c|}
\hline Parameters & Values & Sources \\
\hline$H$ & $0.00125 \mu \mathrm{m}^{3}$ & (Tanner \& Dugger 2003) \\
\hline$D_{1}$ & $1.5 \mathrm{Gpa}$ & (Tanner \& Dugger 2003) \\
\hline$\mu_{\theta}$ & $0.2 \mathrm{Gpa}$ & Assumption \\
\hline$\sigma_{\theta}$ & $0.01 \mathrm{Gpa}$ & Assumption \\
\hline$D_{e}$ & $1.4 \mathrm{Gpa}$ & Assumption \\
\hline$D_{r}$ & $1.2 \mathrm{Gpa}$ & Assumption \\
\hline$\varphi$ & 0 & (Tanner \& Dugger 2003) \\
\hline$\mu_{\beta 1}$ & $8.4823 \times 10^{-9} \mu \mathrm{m}^{3}$ & $\begin{array}{c}\text { (Tanner \& Dugger 2003; } \\
\text { Peng et al. 2011) }\end{array}$ \\
\hline$\sigma_{\beta 1}$ & $6.0016 \times 10^{-10} \mu \mathrm{m}^{3}$ & $\begin{array}{c}\text { (Tanner \& Dugger 2003; } \\
\text { Peng et al. 2011) }\end{array}$ \\
\hline$\mu_{\eta}$ & $2.4823 \times 10^{-9} \mu^{3}$ & Assumption \\
\hline$\sigma_{\eta}$ & $1.0016 \times 10^{-10} \mu \mathrm{m}^{3}$ & Assumption \\
\hline$\lambda$ & $5 \times 10^{-5} /$ revolutions & Assumption \\
\hline$\mu_{W}$ & $1.2 \mathrm{Gpa}$ & Assumption \\
\hline$\sigma_{W}$ & $0.2 \mathrm{Gpa}$ & Assumption \\
\hline$\mu_{Y}$ & $1.0 \times 10^{-4} \mu \mathrm{m}^{3}$ & Assumption \\
\hline$\sigma_{Y}$ & $2 \times 10^{-5} \mu \mathrm{m}^{3}$ & Assumption \\
\hline$\delta$ & $2.0 \times 10^{3}$ revolutions & Assumption \\
\hline$n$ & 2 & Assumption \\
\hline$L$ & 2 & Assumption \\
\hline
\end{tabular}

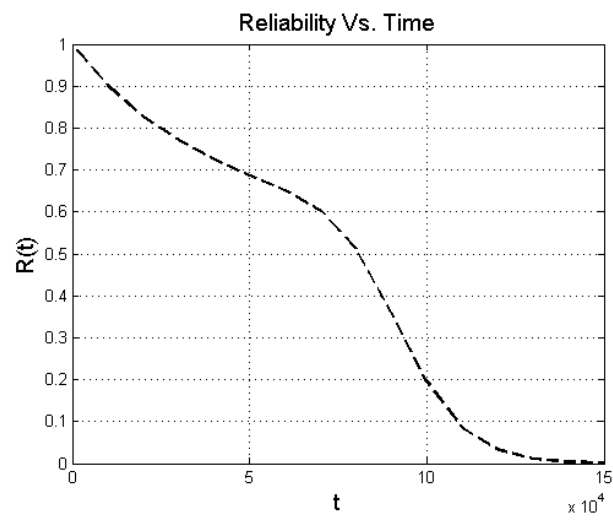

Figure 3: Plot of $R(t)$ 


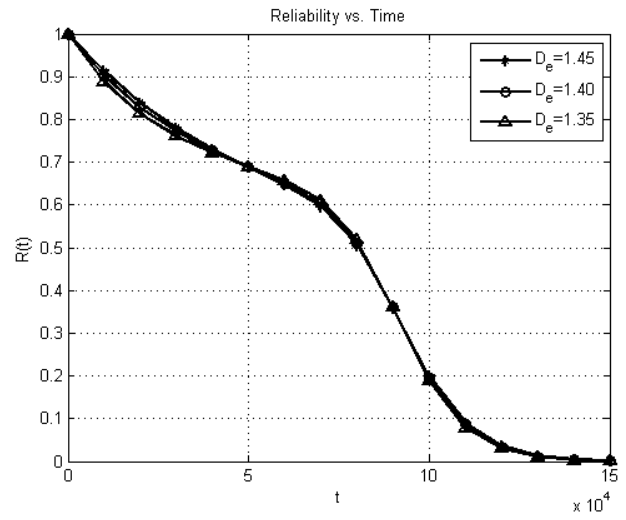

Figure 4: Sensitivity Analysis of $R(t)$ on $D_{e}$

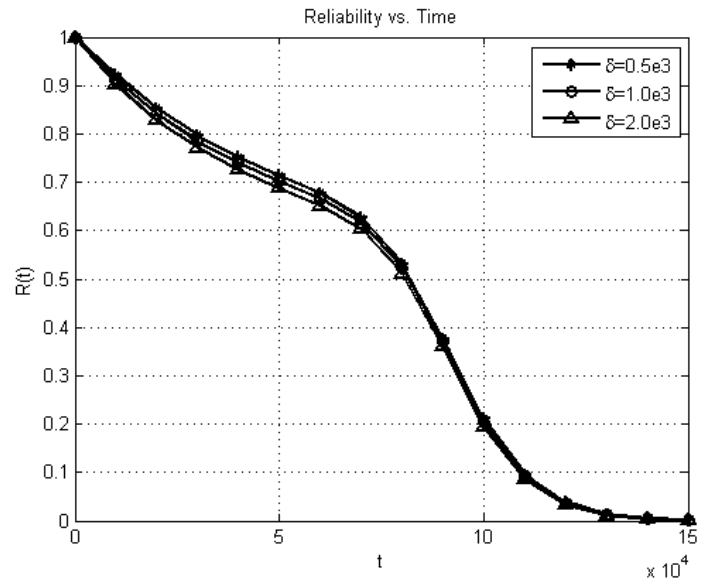

Figure 5: Sensitivity Analysis of $R(t)$ on $\delta$

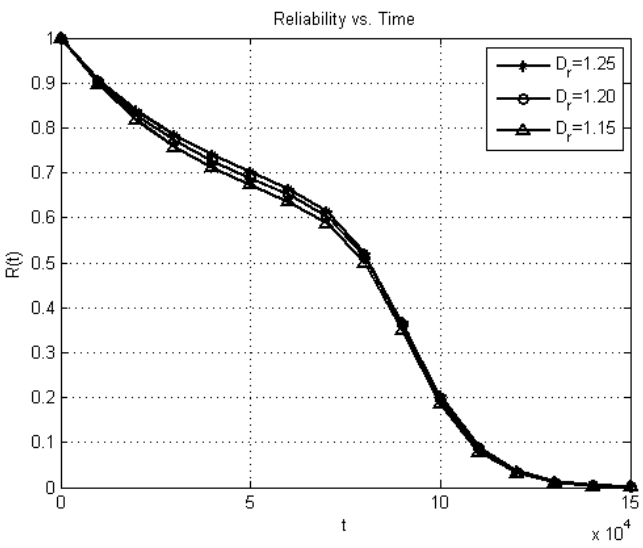

Figure 6: Sensitivity Analysis of $R(t)$ on $D_{r}$ 
As you can see in Figure 4, the critical threshold for extreme shock model $\left(D_{e}\right)$ impacts the reliability of the system by shifting $R(t)$ to the right when $D_{e}$ increases from 1.35 Gpa to 1.45 Gpa. It can be inferred that the reliability of system improves by increasing $D_{e}$.

As can be observed in Figure 5, the time lag threshold $\delta$ affects the reliability function. By increasing $\delta$ from $0.5 \times 10^{3}$ revolutions to $2.0 \times 10^{3}$ revolutions, $R(t)$ shifts slightly to the left. It indicates that the reliability performance is better for the smaller value of $\delta$.

Figure 6 shows that the system reliability is sensitive to the critical threshold for run shock model $\left(D_{r}\right)$. Increasing $D_{r}$ from $1.15 \mathrm{Gpa}$ to $1.25 \mathrm{Gpa}$ causes $R(t)$ to shift to right. When $D_{r}$ approaches the value of $D_{1}, R(t)$ shifts to the right that indicates a more reliable system.

\section{Conclusions and Discussion}

This paper studies a new reliability model for a system subject to dependent competing risks under the impact from a generalized mixed shock model. Two dependent failure risks are soft failure due to degradation process, and hard failure due to shocks. A soft failure occurs when the overall degradation level accumulated by continuous degradation and sudden increment caused by shocks exceeds a predetermined threshold. The hard failure occurs when the size of a fatal shock is beyond the hard failure threshold that can change over time. A device fails as soon as either one of the failure mechanisms occurs. These two failure processes are competing yet dependent, because 1) the shocks damage the unit by increasing the degradation instantaneously, 2) the shock process may speed up the deterioration by accelerating the degradation rate, and 3) the shock process may weaken the unit by reducing the hard failure threshold. While the first impact of nonfatal shocks comes from each individual shocks, the other two impacts are realized when the condition for a generalized mixed shock model is satisfied. The proposed generalized mixed shock model happens if 1) a shock is above a critical value in the extreme shock model, 2) 
a time lag between two sequential shocks is less than $\delta$ in $\delta$-shock model, or 3) a run of $n$ consecutive shocks is greater than a threshold in the run shock model. When the condition for the generalized mixed shock model is satisfied, the degradation rate increases and the hard failure threshold decreases simultaneously.

In this paper, we assume that the effects on the transition (the amount of change in the hard failure threshold/degradation rate) are represented by the same random variables, $\eta_{j}$ and $\theta_{j}$ for different shock models, respectively. The model can be extended to further explore the situation when the occurrence of each shock model produces different effects on the transition, by using different random variables to represent the effects on the transition for different shock models. In the case that their effects on the transition superimpose if two or more shock patterns take place at the same time, it can be modeled by adding the random variables representing the different effects from different shock models.

An example using MEMS devices illustrates the effectiveness of the proposed model with sensitivity analysis, where the values of the parameters are obtained either from the device users or based on some reasonable assumptions. For other device types sharing all or some of the particular features, the general or the special cases can be applied to derive the reliability function. The values of the parameters can be provided from expert knowledge (e.g., material strength governing the initial hard failure threshold) or estimated based on available data (e.g., shock arrival rate). The general reliability function proposed in this paper can assist the decision making in the operation and maintenance of complex systems that are exposed to dependent degradation and shock processes. 
Appendix A: The probability of no hard failure given $S_{1} \leq N(t)=i$

Given that the shock count for the first transition is less than or equal to $N(t), S_{1}=s_{1} \leq$ $N(t)=i$, we have

$$
\begin{aligned}
R_{H}\left(t \mid S_{1}=s_{1} \leq N(t)=i\right)= & \sum_{s_{l}=1}^{I_{l}} \cdots \sum_{s_{2}=1}^{I_{2}} \sum_{l=1}^{L} R_{H}\left(t \mid \bigcap_{j=1}^{l}\left\{S_{j}=s_{j}\right\}, S_{l+1}>I_{l+1}\right) \\
& \times P\left(S_{l+1}>I_{l+1} \mid \bigcap_{j=1}^{l}\left\{S_{j}=s_{j}\right\}\right)\left[\prod_{j=2}^{l} P\left(S_{j}=s_{j} \mid \bigcap_{k=1}^{j-1}\left\{S_{k}=s_{k}\right\}\right)\right] \\
& =\sum_{s_{l}=1}^{i_{l}} \cdots \sum_{s_{2}=1}^{i_{2}} \sum_{l=1}^{L} P\left(\bigcap_{j=1}^{l} \bigcap_{z=1}^{s_{j}}\left\{W_{z}<D_{j}\right\}, \bigcap_{z=1}^{i_{l+1}}\left\{W_{z}<D_{l+1}\right\}\right) \\
& \times P\left(S_{l+1}>i_{l+1}\right)\left[\prod_{j=2}^{l} P\left(S_{j}=s_{j}\right)\right] \\
& =\sum_{s_{l}=1}^{i_{l}} \ldots \sum_{s_{2}=1}^{i_{2}} \sum_{l=1}^{L}\left[\prod_{j=1}^{l} F_{W}\left(D_{j}\right)^{s_{j}}\right] F_{W}\left(D_{l+1}\right)^{i_{l+1}} P\left(S_{l+1}>i_{l+1}\right)\left[\prod_{j=2}^{l} P\left(S_{j}=s_{j}\right)\right] .
\end{aligned}
$$

This formula derives the probability of no hard failure when the system experiences at least one transition by time $t$. The magnitude of each shock must be less than the corresponding hard failure threshold at the time of shock arrival. We use $I_{j+1}=i-\sum_{k=1}^{j} S_{k}$, for $j=1, \ldots, l$, to denote the number of remaining shocks after the $j^{\text {th }}$ transition. To have a total of $l$ transitions, the shock count for the $l+1^{\text {th }}$ transition should be greater than the number of remaining shocks after the $l^{\text {th }}$ transition, $S_{l+1}>I_{l+1}$.

Appendix B: The probability of no soft failure given $S_{1} \leq N(t)=i$

Similar to the derivation in Appendix A, the following derivation takes into account when there is at least one transition by time $t$, i.e., the shock count for the $l+1^{\text {th }}$ transition is greater than the number of remaining shocks after the $l^{\text {th }}$ transition, $S_{l+1}>I_{l+1}$. 


$$
\begin{aligned}
R_{S}\left(t \mid S_{1}=s_{1} \leq N(t)=i\right) & =\sum_{s_{l}=1}^{I_{l}} \cdots \sum_{s_{2}=1}^{I_{2}} \sum_{l=1}^{L} P\left(X_{S}(t)<H \mid \bigcap_{j=1}^{l}\left\{S_{j}=s_{j}\right\}, S_{l+1}>I_{l+1}\right) \\
& \times P\left(S_{l+1}>I_{l+1} \mid \bigcap_{j=1}^{l}\left\{S_{j}=s_{j}\right\}\right)\left[\prod_{j=2}^{l} P\left(S_{j}=s_{j} \mid \bigcap_{k=1}^{j-1}\left\{S_{k}=s_{k}\right\}\right)\right] \\
& =\sum_{s_{l}=1}^{i_{l}} \cdots \sum_{s_{2}=1}^{i_{2}} \sum_{l=1}^{L} P\left(\varphi+\sum_{j=1}^{l} \beta_{j} T_{j}+\beta_{l+1} T_{l+1}+\sum_{z=1}^{i} Y_{z}<H\right) \\
& \times P\left(S_{l+1}>i_{l+1}\right)\left[\prod_{j=2}^{l} P\left(S_{j}=s_{j}\right)\right] .
\end{aligned}
$$

\section{Appendix C: Special Case of $L=1$}

In this case, the probability of no hard failure in Eq. (4), the probability of no soft failure in Eq. (7), and the reliability function in Eq. (10) can be simplified as below.

- When $L=1$, it implies that there is only one transition occurred by time $t$, or at least one shock arrived by time $t$ that meets the condition for the generalized mixed shock model to damage the system. Therefore, the shock count for the first and only transition is less than or equal to $N(t), S_{1} \leq N(t)=i$. The magnitude of each shock before transition must be less than $D_{1}$ or $D_{2}$ (after the transition). The probability of no hard failure given $S_{1} \leq N(t)=i$ is derived to be

$$
R_{H}\left(t \mid S_{1}=s_{1} \leq N(t)=i\right)=F_{W}\left(D_{1}\right)^{s_{1}} F_{W}\left(D_{2}\right)^{i-s_{1}} P\left(S_{2}>i-s_{1}\right) .
$$

- When $L=1$, There is only one shock by time $t$ that meets the condition of the generalized mixed shock model to accelerate the degradation rate. Therefore, the shock count for that trigger shock is less than or equal to $N(t), S_{1} \leq N(t)=i$. The probability of no soft failure given only one degradation rate transition is derived as

$$
R_{S}\left(t \mid S_{1}=s_{1} \leq N(t)=i\right)=P\left(\varphi+\beta_{1} T_{1}+\beta_{2}\left(t-T_{1}\right)+\sum_{z=1}^{i} Y_{z}<H\right) P\left(S_{2}>i_{2}\right) .
$$

- In the special case when $L=1$, the reliability at time $t$ is derived to be 


$$
\begin{aligned}
R(t) & =P\left(\varphi+\beta_{1} t<H\right) \times e^{-\lambda t}+\sum_{i=1}^{\infty} P\left(\varphi+\beta_{1} t+\sum_{z=1}^{i} Y_{z}<H\right) F_{W}\left(D_{1}\right)^{i} P\left(S_{1}>i\right) \frac{e^{-\lambda t}(\lambda t)^{i}}{i !} \\
& +\sum_{i=1}^{\infty} \sum_{s_{1}=1}^{i}\left(\int_{0}^{t} P\left(\varphi+\beta_{1} t_{1}+\beta_{2}\left(t-t_{1}\right)+\sum_{z=1}^{i} Y_{z}<H\right) f_{T_{l}}\left(t_{l}\right) d t_{l}\right) \\
& \times F_{W}\left(D_{1}\right)^{s_{1}} F_{W}\left(D_{2}\right)^{i-s_{1}} P\left(S_{2}>i-s_{1}\right) P\left(S_{1}=s_{1}\right) \frac{e^{-\lambda t}(\lambda t)^{i}}{i !} .
\end{aligned}
$$

\section{Acknowledgments}

The insightful comments from the anonymous reviewers are sincerely appreciated. This research paper was based upon work supported by USA National Science Foundation (NSF) under grants 0970140 and 0969423 . The statements made herein are solely the responsibility of the authors.

\section{Reference}

[1]. Bai, J.-M., Z.-H. Li, and X.-B. Kong. 2006. "Generalized shock models based on a cluster point process." IEEE Transactions on Reliability 55(3): 542-550.

[2]. Chien, Yu-Hung, Shey-Huei Sheu, Zhe George Zhang, and Ernie Love. 2006. "An extended optimal replacement model of systems subject to shocks." European Journal of Operational Research 175(1): 399-412.

[3]. Gut, Allan. 2001. "Mixed shock models." Bernoulli 7(3): 541-555.

[4]. Huynh, K.T., A. Barros, C. Bérenguer, and I.T. Castro. 2011. "A periodic inspection and replacement policy for systems subject to competing failure modes due to degradation and traumatic events." Reliability Engineering \& System Safety 96(4): 497-508.

[5]. Huynh, K.T., I.T. Castro, A. Barros, and C. Bérenguer. 2012. "Modeling age-based maintenance strategies with minimal repairs for systems subject to competing failure modes due to degradation and shocks." European Journal of Operational Research 218(1): $140-151$.

[6]. Jiang, Lei, Qianmei Feng, and David W. Coit. 2012. "Reliability and maintenance modeling for dependent competing failure processes with shifting failure thresholds." IEEE Transactions on Reliability 61(4): 932-948.

[7]. Krieger, Abraham. 1984. "On the probability of n consecutive successes out of $\mathrm{N}$ tries." IEEE Transactions on Aerospace and Electronic Systems AES-20(6): 835-835.

[8]. Lehmann, Axel. 2009. "Joint modeling of degradation and failure time data." Journal of Statistical Planning and Inference 139(5): 1693-1706.

[9]. Li, Chun-yang, Xun Chen, Xiao-shan Yi, and Jun-yong Tao. 2010. "Degraded systems with multiple performance parameters subject to shocks." In Proceedings - Annual Reliability and Maintainability Symposium (RAMS), Ieee, p. 1-6. 
[10]. Li, W., and H. Pham. 2005. "Reliability modeling of multi-state degraded systems with multi-competing failures and random shocks." IEEE Transactions on Reliability 54(2): 297-303.

[11]. Li, Zehui, and Xinbing Kong. 2007. "Life behavior of $\delta$-shock model." Statistics \& Probability Letters 77(6): 577-587.

[12]. Mallor, F., and E. Omey. 2001. "Shocks, runs and random sums." Journal of Applied Probability 38(2): 438-448.

[13]. Peng, Hao, Qianmei Feng, and David W. Coit. 2011. "Reliability and maintenance modeling for systems subject to multiple dependent competing failure processes." IIE Transactions 43(1): 12-22.

[14]. Rafiee, Koosha, Qianmei Feng, and David W. Coit. 2014. "Reliability modeling for dependent competing failure processes with changing degradation rate." IIE Transactions 46(5): 483-496.

[15]. Rafiee, K., Feng, Q., Coit, D.W. "Reliability analysis and condition-based maintenance for multiple failure processes under Degradation-dependent hard failure threshold." Working paper.

[16]. Rafiee, K., Feng, Q., Coit, D.W. 2015. "Condition-based maintenance for repairable deteriorating systems subject to generalized mixed shock model." IEEE Transactions on Reliability, 64(4): 1164-1174.

[17]. Saassouh, B., L. Dieulle, and A. Grall. 2007. "Online maintenance policy for a deteriorating system with random change of mode." Reliability Engineering \& System Safety 92(12): 1677-1685.

[18]. Tanner, D.M., J.a. Walraven, K. Helgesen, L.W. Irwin, F. Brown, N.F. Smith, and N. Masters. 2000. "MEMS reliability in shock environments." In IEEE International Reliability Physics Symposium Proceedings. 38th Annual (Cat. No.00CH37059), Ieee, p. 129-138.

[19]. Tanner, Danelle M, and Michael Dugger. 2003. "Wear mechanisms in a reliability methodology." In Proceedings of SPIE, SPIE, p. 22-40.

[20]. Wang, G. J., and Y. L. Zhang. 2005. "A shock model with two-type failures and optimal replacement policy.” International Journal of Systems Science 36(4): 209-214.

[21]. Wang, Zhong-Lai, Li Du, and Hong-Zhong Huang. 2008. "Reliability modeling for dependent competitive failure processes." In Annual Reliability and Maintainability Symposium, IEEE, p. 278-282.

[22]. Wang, Zhonglai, Hong-Zhong Huang, Yanfeng Li, and Ning-Cong Xiao. 2011. "An approach to reliability assessment under degradation and shock process." IEEE Transactions on Reliability 60(4): 852-863.

[23]. Van der Weide, J.A.M., M.D. Pandey, and J van Noortwijk. 2010. "Discounted cost model for condition-based maintenance optimization." Reliability Engineering \& System Safety 95(3): 236-246.

[24]. Ye, Zhi Sheng, Loon Ching Tang, and Hai Yan Xu. 2011. "A distribution-based systems reliability model under extreme shocks and natural degradation." IEEE Transactions on Reliability 60(1): 246-256.

[25]. Ye, Zhi-Sheng, Yan Shen, and Min Xie. 2012. "Degradation-based burn-in with preventive maintenance." European Journal of Operational Research 221(2): 360-367. 